\title{
Einstein-Kaluza Combined Spacetime as the Optimal and Simplest Framework to Compute and Understand Dark Matter, Pure Dark Energy and Measurable Ordinary Energy
}

\section{Mohamed S. El Naschie}

Department of Physics, Faculty of Science, Alexandria University, Alexandria, Egypt

Correspondence to: Mohamed S. El Naschie, Chaossf@aol.com

Received: July 19, 2017 Accepted: July 24, $2017 \quad$ Published: July 27, 2017

Copyright @ 2017 by authors and Scientific Research Publishing Inc.

This work is licensed under the Creative Commons Attribution International License (CC BY 4.0).

http://creativecommons.org/licenses/by/4.0/

\section{(c) (i) Open Access}

In this rather short communication we hope to draw attention to a new and rather exciting finding which we think represents an important and non-trivial insight into the current dark energy and dark matter cutting edge research [1-11]. In short we found that a combination of the basic invariant of Einstein $D=4$ and Kaluza $D=5$ spacetime manifold is the simplest and optimal setting to perform computation $[7,8]$ and gain insight into the major cosmological problem of dark matter, accelerated cosmic expansion, missing dark energy of the universe [1] as well as the corresponding measurable ordinary energy density which was found, to the surprise of the entire scientific community, to be a mere $4.5 \%$ of what was expected [1,2] based on some of our otherwise most cherished and trusted theories [1-4]. In particular we must stress the importance of the physical insight gained about the source of the difference between the three distinct categories of energy as afforded by the new spacetime picture [7, 8]. Roughly speaking our new conception of spacetime considers a 5 dimensional Kaluza spacetime manifold effectively encasing the 4 dimensional Einstein spacetime [7-9]. In addition we insist on a self similarity [7] of the Einstein interior of the Kaluza spacetime [6] so that in the first iteration, the combined dimension of Kaluza cobordism of Einstein's manifold would be given by the following truncated continued fraction

$$
\begin{aligned}
D & =(\text { EinsteinDimension }+1)+\frac{1}{D(\text { Einstein })}=(4+1)+\frac{1}{4} \\
& =5+\frac{1}{4}=D(\text { Kaluza })+\frac{1}{D(\text { Einstein })}=5.25
\end{aligned}
$$

Letting the iteration go on to infinity, one finds [6]

$$
D=5+\frac{1}{4+\frac{1}{4+\frac{1}{4+\cdots}}}=5+\phi^{3}=5.236067977
$$

where $\phi=(\sqrt{5}-1) 2$ as explained in References $[2,6]$.

This happens to be exactly equal to the dimension corresponding to a fractal Kaluza-Klein spacetime 
considered in numerous previous publications [6]. From the above it becomes an almost trivial guess that ordinary energy density may be estimated to the first approximation to be given by the ratio of the fractal part (i.e. the zero set quantum particle part [6] of the total dimension i.e. $5.25-5.0=0.25$ to the total dimension 5.25. This means:

$$
\gamma(0) \simeq \frac{(1 / 4)}{5+(1 / 4)} \cong 5 \%
$$

Consequently the exact expression must be

$$
\gamma(0)=\phi^{3} /\left(5+\phi^{3}\right)=\frac{1}{22+k}=4.508 \%
$$

Again this is in complete agreement with previous theoretical results [2-4] as well as being in astounding accord with accurate cosmic measurements and observations [1-9].

From the above it is equally obvious to conclude that the dark energy sector must be given by the ratio of the integer part of the Einstein-Kaluza space $D=5$ to the entire dimension i.e. $[6,8]$

$$
\gamma(D)=\frac{5}{5+\phi^{3}} \cong 95.5 \%
$$

This is a remarkable result in view of its complete agreement with that obtained using 'tHooft's Fractal Spacetime namely $[3,4,8]$

$$
\gamma(D)=\frac{4-k}{4}
$$

where $k=\phi^{3}\left(1-\phi^{3}\right)$ is mass charge of 'tHooft's hypothetical renormalon quasi-elementary particle discussed extensively in the literature [3-8].

Now we come to the most remarkable and novel aspect of the present theory or at a minimum the present model namely that $D=5+\phi^{3}$ can be dissected not only into the zero set quantum particle part " $\phi 3$ " which corresponds in fact to five dimensional zero set volume and the empty set quantum wave part " 5 " which corresponds in actuality to a five dimensional empty set volume $[2,4,6]$ but more than that it can be divided into a stringy dark matter energy part $D \simeq 1$ and a 4 -dimensional object part $D \simeq 4$ representing the source of pure dark energy. We hasten to mention here that this part must be a fractal version of 'tHooft's 4 dimensional particle mentioned in [5] i.e. effectively a fractal instanton [3-8]. In fact it will turn out that $D(5)=5$ does not split exactly into

$$
D(5)=D(1)+D(4)=1+4=5
$$

but in the first approximation it splits into a fractal non-integer dimensions given with high accuracy by

$$
D(5)=(1+k)+(4-k)=5
$$

where we see again the profound role played by 'tHooft's renormalon charge $k=0.18033989$ and we conjecture that $4-k$ is nothing but 'tHooft's $4 \mathrm{D}$ particle minus his renormalon particle [3-6].

In other words the source of pure dark energy may be thought of as being a 'tHooft instanton minus a 'tHooft renormalon topological charge of hypothetical quasi particles. In our modest opinion it is only a matter of time until this particle is discovered experimentally.

Before proceeding further let us estimate the dark matter energy density $\gamma(D M)$ and the pure dark energy density $\gamma(P D)$ based on the most rudimentary simple dissection of the 5 dimensional Witten-like $\mathrm{D}=5$ brane bulk [6] into the one dimensional strings and four dimensional objects. This would lead to the obvious densities

$$
\begin{aligned}
& \gamma(D M)=\frac{1}{5.25} \simeq 20 \% \\
& \text { and } \gamma(D D)=\frac{4}{5.25} \simeq 76 \%
\end{aligned}
$$


The above results are by no means accurate but they simply hint at the right direction [1-3] Thus including 'tHooft's renormalon, we are almost home and obtain [8]

$$
\gamma(D M)=\frac{1+k}{5+\phi^{3}} \simeq 0.22=22 \%
$$

and

$$
\gamma(P D)=\frac{4-k}{5+\phi^{3}}=0.7294 \simeq 73 \%
$$

This is of course an excellent approximation obtained by a minimal amount of calculation. To find the exact result, we just need to realize that unlike the ordinary energy sector which is completely decoupled from the dark energy sector, within the dark energy sector itself dark matter and pure dark energy are intricately coupled [8] and quasi higher order renormalons must be taken into account [8]. Thus denoting the coupling term by $\Delta$ we find that it is given by [8]

$$
\Delta=k \frac{\left(8+k^{2}\right) \phi^{3}}{100}=k-0.01896221331=0.1613776767
$$

The exact dark matter energy and pure dark energy densities are consequently given by [8]

$$
\gamma(D M)=\frac{1+\Delta}{5+\phi^{3}}=(22+k) \%
$$

and

$$
\gamma(P D)=\frac{4-\Delta}{5+\phi^{3}}=73.311 \%
$$

in full agreement with the results obtained previously using a far more complex computation [8].

Thus we conclude that Einstein-Kaluza spacetime coupled with 'tHooft's ideas about instanton and renormalon leads to the simplest computation as well as explanations for dark matter, pure dark energy and measurable ordinary energy densities of the cosmos provided, we did in reality cast these ideas into a number system compatible with the golden mean renormalization groups used extensively in non-linear dynamics and deterministic chaos research as done in the present paper [11].

From the above the reader will surely appreciate the fact that scientific knowledge advances like a jigsaw puzzle, step by step and now and then the accumulation of minor enlightenments results in a qualitative large mutation in our perception of Nature, thanks to the dedicated work of people who thought of theories such as Heterotic strings, Brane, Instantons and Renormalons all living in the Einstein and Kaluza-Klein's extra spacetime dimensions [2-9].

\section{REFERENCES}

1. Copeland, E.J., Sami, M. and Tsujikawa, S. (2006) Dynamics of Dark Energy. International Journal of Modern Physics. D, 15, 1753-1935. https://doi.org/10.1142/S021827180600942X

2. El Naschie, M.S. (2013) A Resolution of Cosmic Dark Energy via a Quantum Entanglement Relativity Theory. Journal of Quantum Information Sciences, 3, 23-26. https://doi.org/10.4236/jqis.2013.31006

3. El Naschie, M.S. (2014) On a New Elementary Particle from the Disintegration of the Simplectic 'tHooft-Veltman-Wilson Fractal Spacetime. World Journal of Nuclear Sciences and Technologies, 4, 216-221. https://doi.org/10.4236/wjnst.2014.44027

4. El Naschie, M.S. (2015) Dark Energy and Its Cosmic Density from Einstein's Relativity and Gauge Fields Renormalization Leading to the Possibility of a New 'tHooft Quasi Particle. The Open Astronomy Journal, 8, 1-17. https://doi.org/10.2174/1874381101508010001

5. 'tHooft. G. (1976) Computation of the Quantum Effects Due to a Four-Dimensional Pseudo Particle. Physics 
Review D, 14, 3432-3450. https://doi.org/10.1103/PhysRevD.14.3432

6. El Naschie, M.S. (2016) On a Fractal Version of Witten's M Theory. International Journal of Astronomy and Astrophysics, 6, 135-144. https://doi.org/10.4236/ijaa.2016.62011

7. El Naschie, M.S. (2016) Einstein's Dark Energy via Similarity Equivalence, 'tHooft Dimensional Regularization and Lie Symmetry Groups. International Journal of Astronomy and Astrophysics, 6, 56-81. https://doi.org/10.4236/ijaa.2016.61005

8. El Naschie, M.S. (2017) A Combined Heterotic String and Kähler Manifold Elucidation of Ordinary Energy, Dark Matter, Obber's Paradox and Pure Dark Energy Density of the Cosmos. Journal of Modern Physics, 8, 1101-1118. https://doi.org/10.4236/jmp.2017.87071

9. El Naschie, M.S. (2017) The Looped Light of the Triple-Slit Experiment as a Confirmation for the Extra Dimension of Quantum Spacetime and the Reality of Dark Energy. Optics and Photonics Journal, 7, 19-26. https://doi.org/10.4236/opj.2017.72003

10. El Naschie, M.S. (2017) Spacetime from Zitterbeweguy. Open Journal of Modeling \& Simulation, 5, 169-173. https://doi.org/10.4236/ojmsi.2017.53012

11. Mestel, B.D., Obaldestin, A.H. and Winn, B. (2000) Golden Mean Renormalization for the Harper Equation: The Strong Coupling Fixed Point. Journal of Mathematical Physics, 41, 8304-8330. https://doi.org/10.1063/1.1328743

Submit or recommend next manuscript to SCIRP and we will provide best service for you:

Accepting pre-submission inquiries through Email, Facebook, LinkedIn, Twitter, etc.

A wide selection of journals (inclusive of 9 subjects, more than 200 journals)

Providing 24-hour high-quality service

User-friendly online submission system

Fair and swift peer-review system

Efficient typesetting and proofreading procedure

Display of the result of downloads and visits, as well as the number of cited articles

Maximum dissemination of your research work

Submit your manuscript at: http://papersubmission.scirp.org/

Or contact ns@scirp.org 